\title{
Diagnostic of the Symbiotic Stars Environment by Thomson, Raman and Rayleigh Scattering Processes
}

\author{
M. Sekeráś ${ }^{1}$, A. Skopal ${ }^{1}$ \\ ${ }^{1}$ Astronomical Institute of the Slovak Academy of Sciences, 05960 Tatranská Lomnica, Slovakia
}

Corresponding author: msekeras@ta3.sk

\begin{abstract}
Symbiotic stars are long-period interacting binaries consisting of a cool giant as the donor star and a white dwarf as the acretor. Due to acretion of the material from the giant's stellar wind, the white dwarf becomes very hot and luminous. The circumstellar material partially ionized by the hot star, represents an ideal medium for processes of scattering. To investigate the symbiotic nebula we modeled the wide wings of the resonance lines OVI $\lambda 1032 \AA, \lambda 1038 \AA$ and HeII $\lambda 1640 \AA$ emission line in the spectrum of AG Dra, broadened by Thomson scattering. On the other hand, Raman and Rayleigh scattering arise in the neutral part of the circumstellar matter around the giant and provide a powerful tool to probe e.g. the ionization structure of the symbiotic systems and distribution of the neutral hydrogen atoms in the giant's wind.
\end{abstract}

Keywords: symbiotic stars - spectroscopy.

\section{Introduction}

Symbiotic stars are interacting binary systems consisting of a cool giant and a hot compact star most probably a white dwarf. Typical orbital periods are between 1 and 3 years, but can be significantly larger. A small part of the material lost by the giant in the form of a stellar wind is accreted by the compact star. This process generates a very hot $\left(\mathrm{T}_{h} \sim 10^{5} \mathrm{~K}\right)$ and luminous $\left(\mathrm{L}_{h} \sim 10^{2}-10^{4} \mathrm{~L}_{\odot}\right)$ source of radiation, which then ionizes a fraction of the neutral wind from the giant, giving rise to nebular emission in the so-called symbiotic nebula.

We distinguish two stages of symbiotic systems. A quiescent phase, when the hot star releases its energy with constant rate and spectral energy distribution, and active phase, when the symbiotic system significantly changes its radiation and brightens up by a few magnitudes in the optical.

The partially ionized circumbinary environment represents an ideal medium for scattering processes. In particular we measure effects of the Raman and Rayleigh scattering of the hot star radiation on neutral atoms of hydrogen and the Thomson scattering on free non-relativistic electrons. Fig. 1 shows a sketch of simplified ionization structure of symbiotic stars with location of scattering processes in their environment. In this contribution we present the effects of these three types of scattering processes observed in the spectra of symbiotic stars and their possible applications.

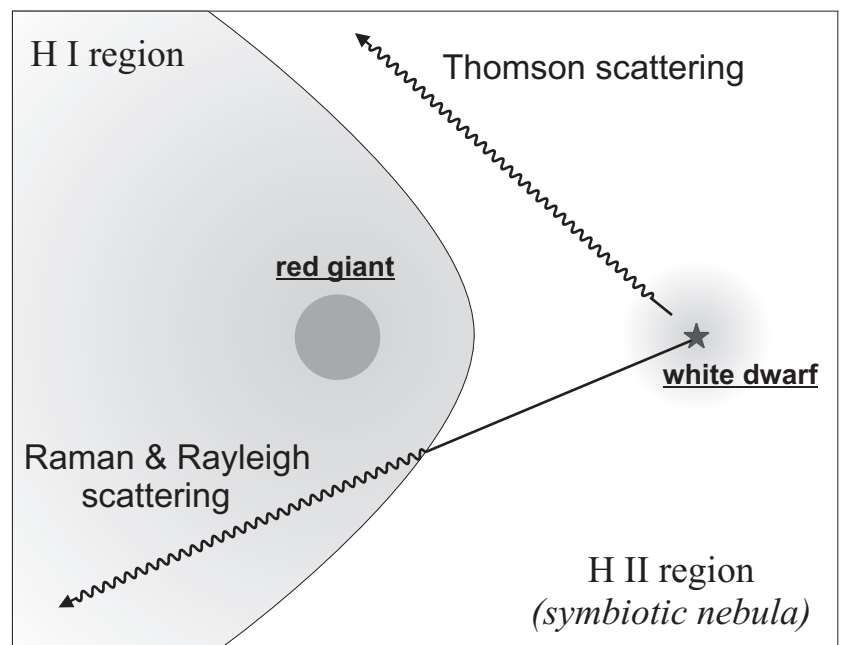

Figure 1: Simplified ionization structure of a symbiotic star. While the radiation from emission region in a vicinity of the white dwarf is Thomson scattered in ionized part of the circumstellar environment, in the neutral part it can be Raman or Rayleigh scattered.

\section{Thomson Scattering}

The scattering of photons on free low-energy electrons $\left(h \nu<<m_{e} c^{2}\right)$ has a very small cross section $\sigma_{T}=6.652 \times 10^{-25} \mathrm{~cm}^{2}$ and is wavelength independent. Within the symbiotic nebula this scattering will attenuate the cores of emission lines redistributing the scattered part of the line into its wings. The wings be- 
Diagnostic of the Symbiotic Stars Environment by Thomson, Raman and Rayleigh Scattering Processes

come very broad, because of a large thermal motions of free electrons, and due to the small value of $\sigma_{T}$, they will be faint. Nevertheless the densest portions of symbiotic nebula with high electron concentration $\left(n_{e} \sim 10^{8}-10^{12} \mathrm{~cm}^{-3}\right)$ together with the extremely intense emission lines of highly ionized elements formed in the vicinity of the hot star (e.g. OVI $\lambda 1032 \AA, \lambda 1038 \AA$ ) allow us to observe these broad wings.

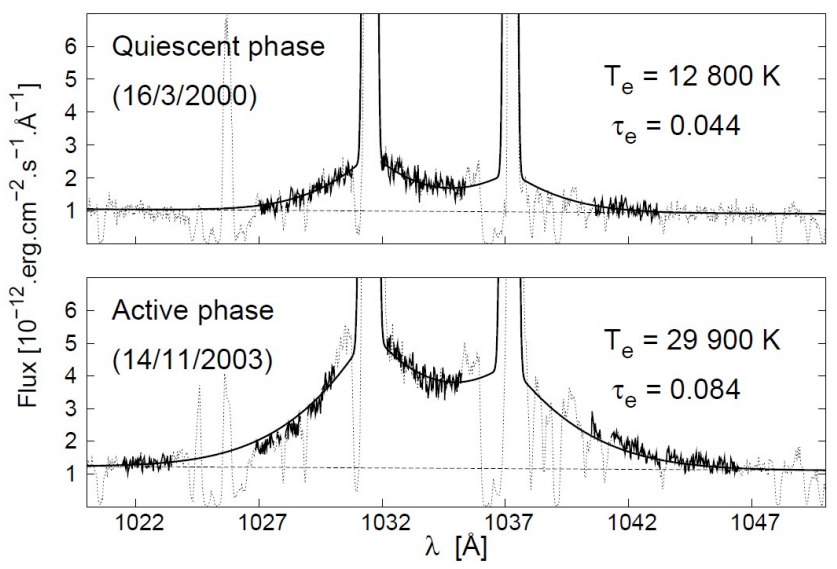

Figure 2: A model (solid line) of the observed (dotted line) profile of the OVI $\lambda 1032, \lambda 1038 \AA$ emission lines as a result of Thomson scattering in the spectra of AG Dra during active and quiescent phase.

To model the broad faint wings of the line profile, we adopted the theoretical line profile used already by Castor et al. (1970). We applied modeling to the resonance dublet OVI $\lambda 1032 \AA, \lambda 1038 \AA$ and HeII $\lambda 1640 \AA$ emission line in the (far-) UV spectra of symbiotic star AG Dra. We determined the mean electron optical depth, $\tau_{e}$, and electron temperature, $T_{e}$, of the symbiotic nebula during different levels of activity (Fig. 2). During quiescent phases the mean value of $\tau_{e}=0.056 \pm 0.006$ and $T_{e}=19200 \pm 2300 \mathrm{~K}$. On the other hand during active phases $\tau_{e}=0.64 \pm 0.11$ and $T_{e}=32300 \pm 2000 \mathrm{~K}$ (see Sekeráš \& Skopal, 2012 in detail).

During the active phases the hot star significantly enhances its stellar wind. This is supported by the broadening of the $\mathrm{H} \alpha$ wings (Skopal, 2006) as well as by the X-ray/UV flux anticorrelation observed in AG Dra during active and quiescent phases (Skopal et al., 2009). The material of the wind is ionized by the luminous hot star, which enhances radiation from the symbiotic nebula, which dominates the spectral region $U$. This produces a significant supplement of free electrons into the nebula. Because $\tau_{e}$ depends on the electron concentration along the line of sight throughout the symbiotic nebula in direction to the hot star, its increase during the active phase indeed indicates the presence of an enhanced mass loss from the white dwarf (Fig. 3).

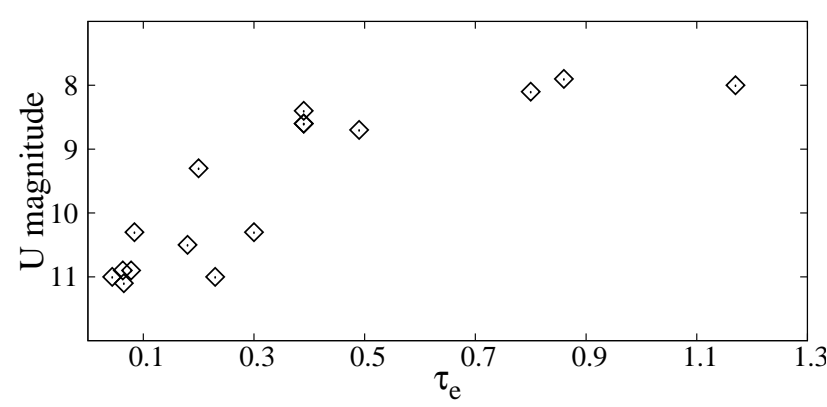

Figure 3: During the transition from the quiescent phase ( $\mathrm{U} \sim 11$; bottom left) to the active phase ( $\mathrm{U} \sim 8$; top right), the ionized enhanced stellar wind from white dwarf represent a new source of free electrons into the nebula, which increases the electron optical depth $\tau_{e}$.

\section{Raman Scattering}

Unlike the Thomson scattering on free electrons, which acts in the ionized part of the red giant's wind (symbiotic nebula), Raman scattering arises within its neutral part around the giant. The Raman scattering on neutral atoms of hydrogen represents the most pronounced effect. It is a process when a photon excites the atom from its ground state to an intermediate state, which is immediately stabilized by a transition to a different lower main energy level, resulting in emitting a photon of a different frequency. We can observe several Raman emission features originated from scattered photons of intense emission lines, such as HeII $\lambda 6545 \AA$ (e.g. Lee et al., 2003), CII $\lambda 7023 \AA, \lambda 7054 \AA$ (Schild \& Schmid, 1996), HeII $\lambda 4851 \AA$ (van Groningen, 1993), etc. However the most conspicuous example of Raman scattering are broad emission lines at $\lambda 6825 \AA$ and $\lambda 7082 \AA$ (e.g. Schmid et al., 1989) (see Fig. 4). These are the characteristic features in the spectrum of about half of symbiotic stars. They are result of scattering of resonance dublet of OVI $\lambda 1032 \AA, \lambda 1038 \AA$ in the far-UV on neutral hydrogen atoms. Their nature was confirmed by near-simultaneous UV and optical observations and spectropolarimetry (e.g. Schild \& Schmid, 1996, Birriel et al., 2000). The Raman scattered lines often show a multi-peaked structure, even when the parental OVI emission line is single-peaked. To explain the line profile differences between the OVI lines and their Raman scattered counterparts, Schmid et al. (1999) suggest Doppler shifts introduced in the Raman scattering process and non-isotropic OVI line emission. Moreover, the evolution of Raman lines during quiescent and active phases can reveal the ionization structure around the hot star (e.g. Skopal et. al., 2006, Fig. 4 here).

One of the main characteristic feature of the Ramanscattered lines is the broadening according to the 
parental UV line. The wavelength $\lambda_{0}$ of Raman scattered radiation is associated with the wavelength $\lambda_{i}$ of the incident UV radiation, which leads to the broadening of the Raman emission lines by factor of $\lambda_{0} / \lambda_{i}$. For example the Raman emission at $6545 \AA$ is broadened according to the parental HeII $\lambda 1025 \AA$ line by a factor of $\approx 6.4$.

As Raman scattering is a dipol process we can observe strong polarization of the broad emission lines $\lambda 6825 \AA$ and $\lambda 7082 \AA$. The amount and orientation of the polarization are expected to change periodically due to the orbital motion. This fact can be helpful to provide information concerning the orbital parameters, such as binary period, orientation and inclination of the orbital plane (e.g. Schild \& Schmid, 1996, Harries \& Howarth, 1996).

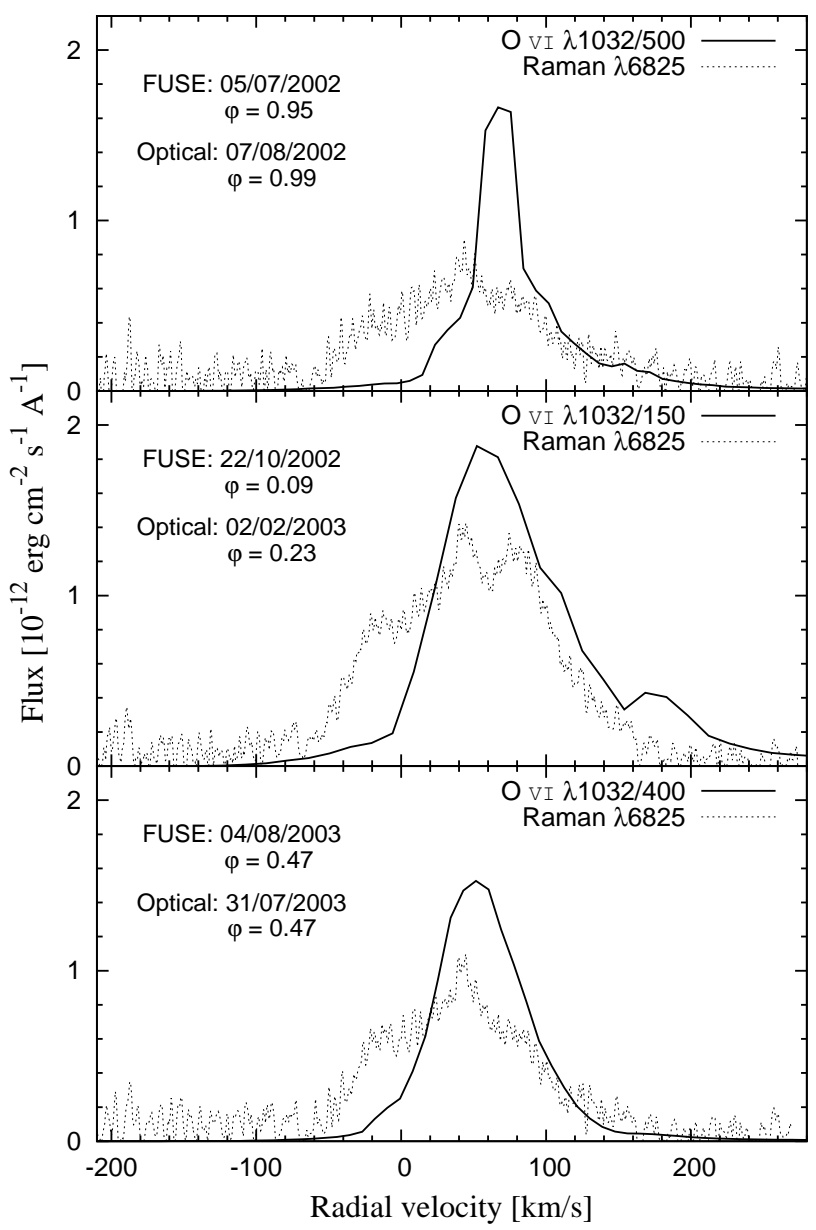

Figure 4: Comparison of the OVI $\lambda 1032 \AA$ line profiles with their Raman scattered counterparts along the outburst in symbiotic star Z And (adopted from Skopal et al., 2006).

In addition to polarimetric measurements, the scattering geometry can be estimated by the Raman scat- tering efficiency. It is defined as the ratio of the number of Raman scattered photons to the number of emitted far-UV photons. This parameter strongly depends on the size of the neutral scattering region as 'seen' by the original photons and thus also on the mass loss rate from the cool giant in symbiotic stars (e.g. Lee et al., 2003).

\section{Rayleigh Scattering}

In the case of Rayleigh scattering, a photon excites an atom from its ground state to an intermediate state, which is immediately stabilized by a transition to a true bound state and this is followed by immediate reemission of a photon of the same wavelength. Thus when the far-UV radiation from/around the hot star goes through the neutral part of the giant's wind, it can be Rayleigh scattered on the neutral hydrogen atoms. Effect of this type of scattering is well observed in the form of strong attenuation of the continuum around hydrogen lines of the Lyman series. The presence of Rayleigh scattering was observed in the spectra of symbiotic stars with a high orbital inclination at/around the position of the inferior conjunction of the giant (e.g. EG And, Vogel, 1991). Figure 5 demonstrates this case for EG And.

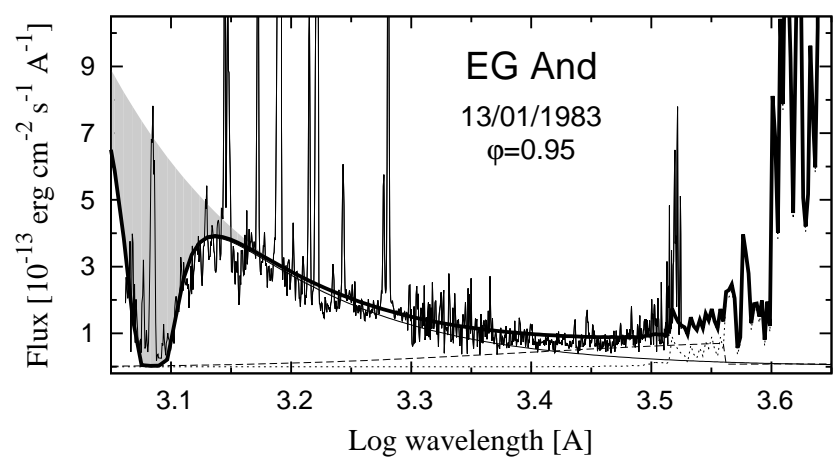

Figure 5: Strong attenuation of the far-UV continuum by Rayleigh scattering process (gray area) in the spectrum of EG And, close to the inferior conjunction of the giant.

The cross section for Raman scattering depends strongly on wavelength with $\lambda^{-4}$, which is well demonstrated by Monte Carlo simulations by Schmid (1995), and increases sharply toward Lyman resonance transitions. Therefore it is easily observable in far-UV spectrum (1200 $\AA-1400 \AA)$ and not distinguishable in the optical. In optically thick environment the photon may undergo many Rayleigh scatterings until it is Raman scattered and escape the neutral region.

Rayleigh scattering arises in the neutral wind around the giant. If we consider the simplified steady- 
Diagnostic of the Symbiotic Stars Environment by Thomson, Raman and Rayleigh Scattering Processes

state ionization model of the symbiotic star (see Fig. 1), where the HI zone is approximately cone-shaped with the giant at its top (Seaquist et al., 1984), it is obvious that the effect of Rayleigh scattering will depend on the ionization structure, the inclination of the orbital plane and the orbital phase. It is therefore most easily measurable in eclipsing systems as periodic changes in the far-UV radiation.

Through the Rayleigh scattering we can measure the neutral hydrogen column density in the line of sight as a function of the orbital phase, with typical values of $10^{20}-10^{24} \mathrm{~cm}^{-2}$. In this way, it is possible to explore the density and distribution of the neutral hydrogen and thus also the velocity structure in outer atmosphere of a red giant (e.g. Vogel, 1991, Pereira et al., 1999).

\section{Conclusions}

The nature of the environment around the symbiotic stars, with a fully ionized and also the neutral part of the circumstellar matter, allow us to investigate symbiotics through the effects of scattering processes.

Thomson scattering on free electrons can diagnose the symbiotic nebula by determining the mean electron optical depth, $\tau_{e}$, and electron temperature, $T_{e}$. While the nebula is directly connected with the changes of the hot star properties, the effects of Thomson scattering can provide some information also about the symbiotic nebula during the quiescent and active phase.

Raman and Rayleigh scattering on neutral hydrogen atoms arises in the neutral part of the giant's wind. They are observed in a form of broad emission features and an attenuation of the far UV continuum, respectively. These processes are useful to examine the ionization structure of the symbiotic systems, kinematics of the scattering and emitting region, the structure and density distribution of the neutral hydrogen in the giant's wind and thus its mass loss rate.

Because the environment, where these scattering processes arises, is a result of the interaction between the giant and the white dwarf, they can provide also information about orbital parameters and physical prop- erties of the two stars independently of other approaches.

\section{Acknowledgement}

The research was supported by a VEGA grant of the Slovak Academy of Sciences No. 2/0002/13.

\section{References}

[1] Birriel, J. J., et al.: 2000, ApJ 545, 1020. doi:10.1086/317851

[2] Castor, J.I., Smith, L.F., van Blerkom, D.: 1970, ApJ 159, 1119. doi:10.1086/150393

[3] Harries, T. J., Howarth, I. D.: 1996, A\&A 310, 235.

[4] Lee, Hee-Won., et al.: 2003, ApJ 598, 553. doi:10.1086/378886

[5] Pereira, C. B., et al.: 1999, A\&A 344, 607.

[6] Schild, H., Schmid, H.M.: 1996, A\&A 310, 221.

[7] Schmid, H. M., et al.: 1989, A\&A 211, L31.

[8] Schmid, H. M., et al.: 1999, A\&A 348, 950.

[9] Seaquist, E. R., Taylor, A. R., Button, S.: 1984, ApJ 284, 202. doi:10.1086/162399

[10] Sekeráš, M., Skopal, A..: 2012, MNRAS 427, 979. doi:10.1111/j.1365-2966.2012.21991.x

[11] Skopal, A.: 2006, A\&A 457, 1003.

[12] Skopal, A., et al.: 2006, A\&A 453, 279.

[13] Skopal, A., et al.: 2009, A\&A 507, 1531.

[14] van Groningen, E.: 1993, MNRAS 264, 975.

[15] Vogel, M.: 1991, A\&A 249, 173. 